\title{
NEURAL NETWORK APPROACH TO PISARENKO'S HARMONIC RETRIEVAL METHOD
}

\author{
George Mathew and V.U. Reddy, \\ Department of Electrical Communication Engineering, \\ Indian Institute of Science, \\ Bangalore 560012
}

ABSTRACT

Pisarenko's Harmonic Retrieval (PHR) method is perhaps the first eigenstructure based spectral estimation technique. The basic step in this method is the computation of the eigenvector corresponding to the minimum eigenvalue of the autocorrelation matrix of the underlying data. This eigenvector is obtained as the solution of a constrained minimization formulation. In this paper, we recast this constrained minimization problem into the neural network (NN) framework by choosing an appropriate cost function (or energy function) for the NN. We also present the theoretical analysis of the proposed approach for the asymptotic case. It is shown that the minimizers of the energy function are the eigenvectors (with a given norm) of the autocorrelation matrix corresponding to the minimum eigenvalue, and vice versa. Further, all the minimizers of this energy function are also global minimizers. Results of computer simulations are presented to support our analysis.

\section{INTRODUCTION}

Estimation of the frequencies of sinusoids corrupted with white noise arises in many applications. The various spectral estimation techniques which can be applied to solve this problem can be classified into two categories; eigenstructure based methods and non-eigenstructure based methods. The Pisarenko's Harmonic Retrieval (PHR) method and the Maximum Entropy Method are the examples, respectively, of these two classes. The eigenstructure based methods are preferrod to the other, since they yield high resolution and asymptotically exact results. In this paper, we concentrate on the PHR method and solve the basic step in this method, i.e., estimation of the eigenvector corresponding to the minimum eigenvalue, using a modified form of the analog Hopfield neural network by exploiting the optimization property of this neural network (NN).

An Artificial Neural Network (ANN) consists of many highly interconnected, simple and similar processing elements, called neurons, operating in parallel. In general, ANNs can be classified into two classes; feedforward ANNs and feedback ANNs. One of the most important applications of the feedback type ANNs is in solving optimization problems. For example, the ability of Hopfield ANN [1] to provide fast and collectively computed solutions to difficult optimization problems is well established in the literature [2],[3],[4].

This paper is organized as follows. The PHR method and its constrained minimization formulation are briefly reviewed in Section 2. The NN formulation of the.PHR problem is presented in Section 3. The theoretical analysis of the proposed approach, derived in Section 3, is presented in Section 4. This includes convergence and such other key aspects. Simulation results are presented in Section 5 and Section 6 concludes the paper.

\section{BACKGROUND}

Let

$$
y(n)=\sum_{i=1}^{P} \alpha_{i} \cos \left(\omega_{i} n+\theta_{i}\right)+v(n)
$$

where $\alpha_{i}, \omega_{i}$ and $\theta_{i}$ denote the amplitude, frequency (normalized) and initial phase (assumed to be uniformly distributed in $[0,2 \pi]$ ) of the $i^{\text {th }}$ sinusoid and $\{v(n)\}$ are zero mean, independent and identically distributed random variables with variance $\sigma^{2}$. Let $\mathbf{R}$ denote the covariance matrix of size $N \times N,(N \geq 2 P+1)$ of $y(n)$. Then the eigenvector corresponding to the minimum eigenvalue (hereafter referred to as the minimum eigenvector) of $\mathbf{R}$ is the solution of the following constrained minimization problem [5]:

$$
\min \quad \mathbf{w}^{T} \mathbf{R w} \quad \text { subject to } \quad \mathbf{w}^{T} \mathbf{w}=1
$$

$\mathbf{w}$

where $\quad \mathbf{w}=\left[w_{1}, w_{2}, \cdots, w_{N}\right]^{T}$, is an N-dimensional weight vector

Now the polynomial whose coefficients are the elements of this minimum eigenvector will have $2 P$ of its $N-1$ roots located at $\exp \left( \pm j \omega_{i}\right), i=1 \ldots P$. These $2 P$ roots of interest will be unaffected by the noise power and the remaining $N-1-2 P$ foots are arbitrary [6].

Thus, the central problem in PHR method is the computation of the minimum eigenvector of the autocorrelation matrix of the underlying data. Different techniques have been recently proposed for efficient and adaptive estimation of the minimum eigenvector [5],[7]. While Thompson [5] suggested a constrained gradient search procedure, Reddy et al. [7] restated this constrained minimization problem as an unconstrained minimization problem and developed a Gauss-Newton type recursive algorithm, for seeking the minimum eigenvector. Larimore [8] studied the convergence behaviour of the Thompson's [5] adaptive algorithm.

In this paper, we suggest a neural network (NN) approach to the PHR problem. We recast the constrained minimization problem (2.2) into a different unconstrained minimization problem, suitable for the NN framework. The analysis and results presented in this paper are for the asymptotic case. However, this approach can easily be extended to the finite data case to develop an adaptive version of the PHR method.

\section{NN FORMULATION OF THE PHR PROBLEM}

In a Hopfield NN, the neurons are connected in a feedback configuration and the stable stationary states of the network correspond to the minima of a mathematical quantity, called as the energy function (or Lyapunov function) of the network. In order to set up a Hopfield NN to minimize a given cost function, the neuron model is fixed a-priori and the connection strengths of the 
neurons are assigned appropriate values by comparing the energy function and the given cost function. But, in the approach we discuss below, the neuron model (and hence the network structure) evolves as a direct consequence of the nature of the cost function to be minimized. In the following derivation, we assume linear input-output relation for each neuron and the output of the $k^{\text {th }}$ neuron represents the $k^{\text {th }}$ element of the vector $\mathrm{w}$.

The cost function used in our development is motivated as follows. Recall that in solving the minimization problem (2.2) using the Lagrange multiplier approach, we use the following cost function

$$
L(\mathbf{w}, \lambda)=\mathbf{w}^{T} \mathbf{R} \mathbf{w}+\lambda\left(\mathbf{w}^{T} \mathbf{w}-1\right)
$$

where $\lambda$ is the Lagrange multiplier. This function is not always positive definite, and hence, it is not a valid energy function (Lyapunov function). We, therefore, modify the second term in (3.1) and construct another function $J$ given by

$$
J(\mathbf{w}, \mu)=\mathbf{w}^{T} \mathbf{R} \mathbf{w}+\mu\left(\mathbf{w}^{T} \mathbf{w}-1\right)^{2}
$$

where $\mu$ is a positive constant. Since $\mathbf{R}$ is a positive definite symmetric matrix (for the case of sinusoids in white noise) and $\mu$ is positive, the function $J$ is always positive. Thus, (3.2) is a valid energy function. Incidentally, the $\mu$ here acts as a weighting given to the violation of the unit norm constraint on the minimizer of $J$.

Now, to obtain the structure of the neural network which solves the minimization problem (3.2), we proceed as below using the Lyapunov stability approach. We can accept $J$ as the energy (or Lyapunov) function for the network to be obtained, provided the network dynamics are such that the time derivative of $J$ is negative.

The time derivative of $J$ is given by

$$
\frac{d J}{d t}=\sum_{k=1}^{N} \frac{\partial J}{\partial w_{k}(t)} \cdot \frac{d w_{k}(t)}{d t}
$$

where $w_{k}(t)$ denotes the $k^{\text {th }}$ component of the vector $\mathbf{w}$ at time $t$ ( $t$ denotes continuous time) and

$$
\cdot \frac{\partial J}{\partial w_{k}(t)}=2 \sum_{p=1}^{N} R_{k p} w_{p}(t)+4 \mu w_{k}(t)\left(\mathbf{w}^{T}(t) \mathbf{w}(t)-1\right)
$$

with $R_{p k}$ denoting the $(p, k)^{t h}$ element of the matrix $\mathbf{R}$. Now, suppose we define the dynamics of the $k^{\text {th }}$ neuron as

$$
\begin{aligned}
\frac{d w_{k}(t)}{d t} & =-\frac{\partial J}{\partial w_{k}(t)} \\
& =-2 \sum_{p=1}^{N} R_{k p} w_{p}(t)-4 \mu w_{k}(t)\left(\mathbf{w}^{T}(t) \mathbf{w}(t)-1\right) \\
k & =1 \ldots N
\end{aligned}
$$

giving

$$
\frac{d J}{d t}=-\sum_{k=1}^{N}\left(\frac{d w_{k}(t)}{d t}\right)^{2}
$$

We note from (3.6) that

$$
\begin{array}{lll}
\frac{d J}{d t}<0 & \text { if } \quad \frac{d w_{k}(t)}{d t} \neq 0 & \text { for at least one of the k's } \\
\frac{d J}{d t}=0 & \text { if } \quad \frac{d w_{k}(t)}{d t}=0 & \text { for all } \mathrm{k} .
\end{array}
$$

This implies that the NN with dynamics given by (3.5) has its stable stationary points at the local minima of $J$. In the next section, we show that the minimizer of $J$ corresponds to a minimum eigenvector of $\mathbf{R}$ (see Theorem 2, Section 4.1). Hence, we can say that when the network comes to the resting state, the neuronal output vector $\mathrm{w}$ will correspond to a minimum eigenvector of $\mathbf{R}$.

For the purpose of illustration, we have shown in Fig. 1 the structure of the resulting $N N$ for the case with $N=3$ and $P=1$. We call this a modified form of the Hopfield NN due to its similarity with the original analog Hopfield NN [1].

\section{THEORETICAL ANALYSIS OF THE PROPOSED APPROACH}

In this section, we present a theoretical analysis of the proposed neural network approach and establish it as a minimum eigenvector estimator. We do this in two steps. First, we establish the correspondence between the minimizers of $J$ and the minimum eigenvectors of $\mathbf{R}$. Next, we derive the bounds on the integration time-step $(h)$ which is used in solving numerically the system of $N$ ordinary differential equations.

We treat the problem of minimization of $J$ as an unconstrained non-linear optimization problem in the following analysis. We study the nature of the stationary points of $J$ (points at which the derivative of $J$ with respect to $\mathbf{w}$ becomes zero) and establish the relationship between these points and the eigenvectors of $\mathbf{R}$. Going one step further, we investigate the link between the minimizers of $J$ and the minimum eigenvectors of $\mathbf{R}$, since our ultimate aim is to solve for a minimum eigenvector of $\mathbf{R}$.

The cost function $J$, as given by (3.2), can be considered as a function of $w$ with parameter $\mu$. In the following analysis, we assume that $\mu$ is fixed at some appropriately chosen value. Guidelines for choosing the value of $\mu$ are given in the discussion that follows Corollary 4.

\subsection{Correspondence Between the Minimizers of $J$ and the Minimum Eigenvectors of $\mathbf{R}$}

In this subsection, we establish the correspondence between the minimizers of $J$ and the minimum eigenvectors of $\mathbf{R}$. The proposition, theorems and corollaries which are stated below are proved in [9].

Positivity assumption on $\mu$ that

In the foregoing analysis, we make the following assumption

$$
\mu>0
$$

This assumption is required to ensure the positivity of $J$.

Proposition: If $w$ is a stationary point (SP) of $J$, then the norm of $\mathbf{w}, \beta$, is less than unity, i.e., $\|\mathbf{w}\|_{2}=\beta<1$, where $\|\cdot\|_{2}$ denotes the Euclidean norm.

Theorem 1: $w$ is a stationary point (SP) of $J$ if and only if $\mathbf{w}$ is an eigenvector of $\mathbf{R}$ corresponding to the eigenvalue $\lambda$, with $\|w\|_{2}^{2}=\beta^{2}=1-\frac{\lambda}{2 \mu}$.

Theorem 2: $\quad w$ is a global minimizer of $J$ if and only if $w$ is a minimum eigenvector of $\mathbf{R}$ corresponding to the minimum eigenvalue $\lambda_{\min }$, with $\|w\|_{2}^{2}=\beta^{2}=1-\frac{\lambda_{\min }}{2 \mu}$.

We state four corollaries below in order to bring out the significant features of Theorem 2.

Corollary 1: The value of $\mu$ should be such that $\mu>\frac{\lambda_{\min }}{2}$.

Corollary 2: For a given $\mu$, every local minimizer of $J$ is also a global minimizer and the minimum value of $J$ is $\frac{\lambda_{\text {min }}}{2}\left(1+\beta^{2}\right)$. Corollary 3: The minimizer of $J$ is unique only when $N=$ 
$2 P+1$.

Corollary 4: The eigenvectors of $\mathbf{R}$ associated with the first

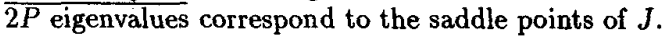

\section{Discussion:}

The proposition implies that the set of stationary points of $J$ with $\mu>0$ is a subset of the set of vectors with norm less than unity. Theorems 1 and 2 establish the one-to-one correspondence between the minimizers of $J$ and the minimum eigenvectors of $\mathbf{R}$. Theorem 1 implies that all the stationary points of $J$ are eigenvectors of $\mathbf{R}$ with a given norm, where this norm is decided by the value of $\mu$. Conversely, all eigenvectors of $\mathbf{R}$. with a given norm are stationary points of $J$. Similarly, Theorem 2 along with Corollary 2 establishes the fact that all minimizers of $J$ are minimum eigenvectors of $\mathbf{R}$, with a norm decided by the value of $\mu$, and vice versa. Corollary 4 reinforces this fact by showing that all other eigenvectors of $\mathbf{R}$ correspond to the saddle points of $J$. Combining these four points we see that computing a minimum eigenvector of $R$ is equivalent to finding a minimizer of $J$. It is significant to note that eventhough $J$ is a non-convex nonlinear function, the problem of minimization of $J$ doesnot suffer from local minima problems since any locally optimum solution is also globally optimum (Corollary 2).

An important point that is to be noted from Theorem 2 is that the norm of all minimizers of $J$ is predetermined by the value of $\mu$. Further, for the constraint satisfaction to be better (i.e., for the norm of the solution to be closer to unity), the value of $\mu$ required is higher. If the minimum eigenvalue of $\mathbf{R}$ is known, then we can choose the value of $\mu$ so as to obtain a minimum eigenvector with a specified norm (less than unity).

\subsection{Bounds on the Integration Time-step}

We note from (3.5) that the vector differential equation, which defines the evolution of the neural network in its state space, is given by

$$
\frac{d \mathbf{w}(t)}{d t}=-2 \mathbf{R} \mathbf{w}(t)-4 \mu \mathbf{w}(t)\left[\mathbf{w}^{T}(t) \mathbf{w}(t)-1\right]
$$

Minimizer of $J$ corresponds to the solution of this vector differential equation. In order to solve this using some numerical technique, we need to choose an appropriate integration time-step, say $h$. The choice of $h$ is crucial, from the point of view of convergence of the technique to the correct solution. We now present an approximate analysis to obtain the upper and lower bounds for $h$, assuming a simple time-discretization numerical technique.

For sufficiently small $h$, we have the following approximation

$$
\left.\frac{d \mathbf{w}(t)}{d t}\right|_{t=n h} \approx \frac{\mathbf{w}(n+1)-\mathbf{w}(n)}{h}
$$

where $n$ is the discrete time index. Thus, (4.1) can be rewritten as

$$
\mathbf{w}(n+1) \approx \mathbf{B}(n) \mathbf{w}(n)
$$

where $\mathbf{B}(n)=[1-4 h \mu d(n)] \mathbf{I}_{N}-2 h \mathbf{R}$ and $d(n)=$ $\mathbf{w}^{T}(n) \mathbf{w}(n)-1$. Noting that $\mathbf{B}(\mathrm{n})$ is symmetric and the eigenvectors of $\mathbf{B}(\mathbf{n})$ are same as those of $\mathbf{R}$, we can express (4.2) as

$$
\mathbf{w}(n+1) \approx \sum_{i=1}^{N} b_{i}(n) \mathbf{e}_{i} \mathbf{e}_{i}^{r} \mathbf{w}(n)
$$

where $\mathbf{e}_{i}$ is the normalized eigenvector corresponding to the eigenvalue $b_{i}(n)\left(=1-4 h \mu d(n)-2 h \lambda_{i}\right)$ of $\mathbf{B}(n)$ and $\lambda_{i}$ is the eigenvalue of $\mathbf{R}$ corresponding to $\mathbf{e}_{\boldsymbol{i}}$.
Let $M_{0}$ be a large positive integer such that for $n>M_{0}$, the trial solution $\mathbf{w}(n)$ is very close to the desired solution, say $w^{*}$. Then it is reasonable to assume that the norm of $w(n)$ remains constant at $\left\|w^{*}\right\|_{2}$, for all practical purposes, from instant to instant. Thus, for $n>M_{\text {。 }}$

$$
\begin{aligned}
d(n) & \approx d=\mathbf{w}^{*} \mathbf{w}^{*}-1 \\
b_{i}(n) \approx b_{i} & =1-4 h \mu d-2 h \lambda_{i}
\end{aligned}
$$

Substituting these approximations into (4.3) and iterating it from $M_{\text {o to }} n$, we can show that

$$
\mathrm{w}(n) \approx \sum_{i=1}^{N} b_{i}^{\left(n-M_{0}\right)} \gamma_{i} \mathbf{e}_{i}
$$

where $\gamma_{i}=\mathrm{e}_{i}^{T} \mathbf{w}\left(M_{0}\right)$. Decomposing (4.5) into two terms, one consisting of the first $2 P$ eigenvalues and eigenvectors and the other consisting of the last $N-2 P$ minimum eigenvalues and eigenvectors (of $\mathbf{R}$ ), we have

$$
\mathrm{w}(n) \approx \sum_{i=1}^{2 P} b_{i}^{\left(n-M_{0}\right)} \gamma_{i} \mathbf{e}_{i}+\sum_{i=2 P+1}^{N} b_{i}^{\left(n-M_{0}\right)} \gamma_{i} \mathbf{e}_{i}
$$

We note from (4.6) that for $\mathbf{w}(\mathbf{n})$ to converge to a minimum eigenvector of $\mathbf{R}$, the first term should vanish asymptotically and the factor $b_{i}^{n-M_{0}}$ in the second term should be a constant for all $n>M_{\text {o }}$. These requirements are met if

$$
\left|b_{i}\right|<1 \quad \forall i=1, \ldots, 2 P
$$

and

$$
\left|b_{i}\right|=1 \quad \forall i=2 P+1, \ldots, N .
$$

Substituting (4.4) into (4.7) and rearranging the terms, we get

$$
0<h<\frac{1}{2 \mu d+\lambda_{i}} \quad \forall i=1, \ldots, 2 P
$$

Since the bound on $h$ has to be satisfied for all eigenvalues, $\lambda_{1}$ to $\lambda_{2 P}$, we replace $\lambda_{i}$ in (4.9) with $\lambda_{\max }$ and restate the relation for $h$ as

$$
0<h<\frac{1}{2 \mu d+\lambda_{\max }}
$$

where $\lambda_{\text {max }}$ is the maximum eigenvalue of $\mathbf{R}$.

Suppose that the eigenvalues of $\mathbf{R}$ are ordered as

$$
\lambda_{1} \geq \lambda_{2} \geq \cdots \geq \lambda_{2 P}>\lambda_{2 P+1}=\lambda_{2 P+2}=\cdots=\lambda_{N}
$$

Then, (4.4) shows that

$$
b_{1} \leq b_{2} \leq \cdots \leq b_{2 P}<b_{2 P+1}=b_{2 P+2}=\cdots=b_{N}
$$

Then, combining (4.7) with (4.11), gives

$$
b_{i}>-1 \quad \forall i \in\{2 P+1, \ldots, N\}
$$

Equations (4.8) and (4.12) imply that for $i=2 P+1, \ldots, N$, .

$$
b_{i}=1-4 h \mu d-2 h \lambda_{i}=1
$$

Substituting for $\lambda_{i}=\lambda_{\min }$ and $d=\mathbf{w}^{* T} \mathbf{w}^{*}-1=\beta^{2}-1$ into (4.13) gives

$$
\lambda_{\min }=2 \mu\left(1-\beta^{2}\right)
$$

Note that (4.14) is in agreement with Theorem 2. Substituting (4.14) into (4.10), we get the bounds on $h$ as

$$
0<h<\frac{1}{\lambda_{\max }-\lambda_{\min }}
$$


Summarizing the analysis of Sections 4.1 and 4.2, we have the following results.

1. All the minimum eigenvectors of $\mathbf{R}$ estimated using the above approach will have norm less than unity.

2. $\mathbf{w}$ is a minimizer of $J$ if and only if it is a minimum eigenvector of $\mathbf{R}$ with $\mathbf{w}^{T} \mathbf{w}=1-\frac{\lambda_{\min }}{2 \mu}$, where $\lambda_{\min }$ is the minimum eigenvalue of $\mathbf{R}$.

3. As the value of $\mu$ increases, the norm of the solution vector approaches unity.

4. The value chosen for $\mu$ should always be greater than $\frac{\lambda_{\text {min }}}{2}$ in order to obtain a valid solution. For $\mu<\frac{\lambda_{\text {min }}}{2}$ the nature of the solution is unpredictable.

5. For a given $\mu$, all minimizers of $J$ (or minimum eigenvectors of $\mathbf{R}$, the norms of which satisfy the relation given above) are global minimizers.

6. The bounds on the integration time-step $h$, for the iterative equation in $w(n)$ to converge to a minimum eigenvector of $\mathbf{R}$, are

$$
0<h<\frac{1}{\lambda_{\max }-\lambda_{\min }}
$$

where $\lambda_{\max }$ is the maximum eigenvalue of $\mathbf{R}$.

This completes the analysis of the proposed neural network approach. Next we present some simulation results which corroborate our analysis.

\section{SIMULATION RESULTS}

For the data described by (2.1), the asymptotic autocorrelation matrix $\mathbf{R}$ is given by

$$
R(i, j)=\sum_{\ell=1}^{P} \frac{\alpha_{\ell}^{2}}{2} \cos \left(\omega_{\ell} k\right)+\sigma^{2} \delta_{k 0}
$$

where $k=|i-j|, \quad i, j=1, \ldots, N$, and $\delta_{k 0}$ is the Kronecker delta function. The system of $N$ ordinary non-linear differential equations is solved numerically, with an appropriate integration timestep $h$. The iterations are stopped when the norm of the difference between the consecutive solution vectors is less than a predetermined threshold, $\delta\left(i . e .,\|\mathbf{w}(n+1)-\mathbf{w}(n)\|_{2}<\delta\right)$. A polynomial, whose coefficients are the elements of the minimum eigenvector estimated using this approach, is then formed and the frequencies of the sinusoids are computed from the roots of this polynomial which are closest to the unit circle. If $\hat{w}$ denotes the estimated minimum eigenvector, then the minimum eigenvalue is estimated as .

$$
\hat{\lambda}_{\min }=\frac{\hat{\mathbf{w}}^{T} \mathbf{R} \hat{\mathbf{w}}}{\hat{\mathbf{w}}^{T} \hat{\mathbf{w}}}
$$

and $\|\hat{w}\|_{2}$ is taken as the estimate of $\beta$.

In the simulations, we chose $\sigma^{2}=1$ (giving $\lambda_{\min }=1$ ) and $\delta=10^{-6}$. For a fixed $h$, the estimated values of the frequencies of the sinusoids, $\hat{\lambda}_{\min }$ and $\hat{\beta}$ are given in Table 1 , for different values of $N, P$ and $\mu$. We note the following from the results of this table.

When $\mu$ is large, $\hat{\beta}$ is closer to unity as predicted by the cost function (3.2). The estimated value of $\lambda_{\min }$ is same as the true value and the norm of the solution vector, $\hat{\beta}$, is very close to the theoretical value given by (4.14). The minor deviations of the estimated values of the frequencies from the true values are because of the numerical solution of the differential equations.

Table 2 gives six different minimizers of $J$ (minimum eigenvectors of $\mathbf{R}$ ) obtained with different initial conditions, for the case with $N=4, P=1$ and $\mu=15$. Note that there are more than $N-2 P$ different minimizers (all having the same norm) thus illustrating the non-uniqueness of the minimizer of $J$, when $N>2 P+1$ (Corollary 3). The estimate of $\lambda_{\min }$ and the norm of the solution vectors are same as the true values. For $\mu \leq \frac{\lambda_{\min }}{2}$, the behaviour of the system was erroneous.

Thus, the simulation results confirm the theoretical assertions we made in Section 4.

\section{CONCLUSIONS}

The problem of estimating the frequencies of a given number of real sinusoids corrupted with white noise using the Pisarenko's harmonic retrieval method has been recast into the neural network framework. Dynamics of the neural network are derived using the Lyapunov stability approach. The theoretical analysis of convergence and other key aspects is developed, and the results of the analysis are supported by simulations. Though we considered the asymptotic case in the paper, the approach can be easily extended to the finite data case.

\section{References}

[1] J. J. Hopfield, "Neurons with Graded Response have Collective Computational Properties like those of two-state Neurons," Proc. Natl. Acad. Sci., USA, vol.81, pp.3088-3092, May 1984.

[2] J. J. Hopfield and D. W. Tank, "Neural Computations of Decisions in Optimization Problems," Biological Cybernatics, vol.52, pp.1411- 152, 1985.

[3] J. J. Hopfield and D. W. Tank, "Computing with Neural Circuits: A Model," Science, vol.233, pp.625-633, Aug. 1986.

[4] D. W. Tank and J. J. Hopfield, "Simple 'Neural' Optimization Networks: An A/D Converter, Signal Decision Circuit and a Linear Programming Circuit," IEEE Trans. Circuits and Systems, vol.CAS-33, pp.533-541, May 1986.

[5] P. A. Thompson, "An Adaptive Spectral Analysis Technique for Unbiased Frequency Estimation in the Presence of White Noise," in Proc. $13^{\text {th }}$ Asilomar Conf. Circiuts, Syst., Computers, Pacific Grove, CA, pp.529-533, Nov.1979.

[6] V. F. Pisarenko, "The Retrieval of Harmonics by a Covariance Function," Geophys. J. Roy. Astron. Soc., pp.347-366, 1973.

[7] V. U. Reddy, B. Egardt and T. Kailath, "Least Squares Type Algorithm for Ádaptive Implementation of Pisarenko's Harmonic Retrieval Method," IEEE Trans. Acoust., Speech, Signal Processing, vol.ASSP-30, pp.399- 405, June 1982.

[8] M. G. Larimore, "Adaptive Convergence of Spectral Estimation based on Pisarenko's Harmonic Retrieval," IEEE Trans. Acoust., Speech, Signal Processing, vol.ASSP-31, pp.955-962, Aug. 1983.

[9] G. Mathew and V. U. Reddy, "Development and Analysis of A Neural Network Approach to Pisarenko's Harmonic Retrieval Method," Submitted to IEEE Trans. Acoust., Speech, Signal Processing. 
Table 1. Estimates of frequencies, $\beta$ and $\lambda_{\min }$ for different values of $\mu, N$ and $P$.

\begin{tabular}{|c|c|c|c|c|c|c|c|c|c|c|}
\hline \multirow[b]{2}{*}{$\mu$} & \multirow[b]{2}{*}{$\mathrm{N}$} & \multirow[b]{2}{*}{$\mathbf{P}$} & \multicolumn{3}{|c|}{ True frequencies } & \multicolumn{3}{|c|}{ Estimated frequencies } & \multirow[b]{2}{*}{$\hat{\beta}$} & \multirow[b]{2}{*}{$\hat{\lambda}_{\min }$} \\
\hline & & & $f_{1}$ & $f_{2}$ & $f_{3}$ & $\hat{f}_{1}$ & $\tilde{f}_{2}$ & $\hat{f}_{3}$ & & \\
\hline 1 & 5 & 1 & 0.20 & & & 0.2000 & & & 0.707356 & 1.00 \\
\hline 40 & 5 & 1 & 0.20 & & & 0.2003 & & & 0.993730 & 1.00 \\
\hline 1 & 5 & 2 & 0.20 & 0.24 & & 0.2003 & 0.2402 & & 0.707106 & 1.00 \\
\hline 40 & 5 & 2 & 0.20 & 0.24 & & 0.2002 & 0.2402 & & 0.993730 & 1.00 \\
\hline 1 & 7 & 1 & 0.20 & & & 0.2000 & & & 0.707355 & 1.00 \\
\hline 40 & 7 & 1 & 0.20 & & & 0.2000 & & & 0.993730 & 1.00 \\
\hline 1 & 7 & 2 & 0.20 & 0.24 & & 0.2001 & 0.2401 & & 0.707100 & 1.00 \\
\hline 40 & 7 & 2 & 0.20 & 0.24 & & 0.2000 & 0.2400 & & 0.993730 & 1.00 \\
\hline 1 & 9 & 1 & 0.20 & & & 0.2000 & & & 0.707356 & 1.00 \\
\hline 40 & 9 & 1 & 0.20 & & & 0.2000 & & & 0.993730 & 1.00 \\
\hline 1 & 9 & 2 & 0.20 & 0.24 & & 0.2000 & 0.2400 & & 0.706865 & 1.00 \\
\hline 40 & 9 & 2 & 0.20 & 0.24 & & 0.2000 & 0.2400 & & 0.993730 & 1.00 \\
\hline 1 & 9 & 3 & 0.20 & 0.24 & 0.28 & 0.2001 & 0.2404 & 0.2801 & 0.707106 & 1.00 \\
\hline 40 & 9 & 3 & 0.20 & 0.24 & 0.28 & 0.2001 & 0.2403 & 0.2801 & 0.993730 & 1.00 \\
\hline
\end{tabular}

True value of $\beta: 0.707106$ for $\mu=1$ and 0.993730 for $\mu=40$

Table 2. Different minimizers (minimum eigenvectors) for the case with $N=4, P=1$ and $\mu=15$.

\begin{tabular}{|c|r|r|r|r|r|r|}
\hline Minimum & 0.7452 & 0.3003 & 0.7190 & 0.6495 & 0.1777 & 0.3051 \\
eigenvector & -0.0739 & 0.5533 & 0.0962 & 0.2481 & 0.6011 & -0.6115 \\
for different & 0.5062 & -0.1564 & 0.3849 & 0.2481 & -0.2616 & 0.5665 \\
initial conditions & 0.3867 & 0.7389 & 0.5406 & 0.6495 & 0.7109 & -0.4229 \\
\hline Norm & 0.983192 & 0.983192 & 0.983192 & 0.983192 & 0.983192 & 0.983192 \\
\hline
\end{tabular}

True value of $\beta=0.983192$ for $\mu=15$

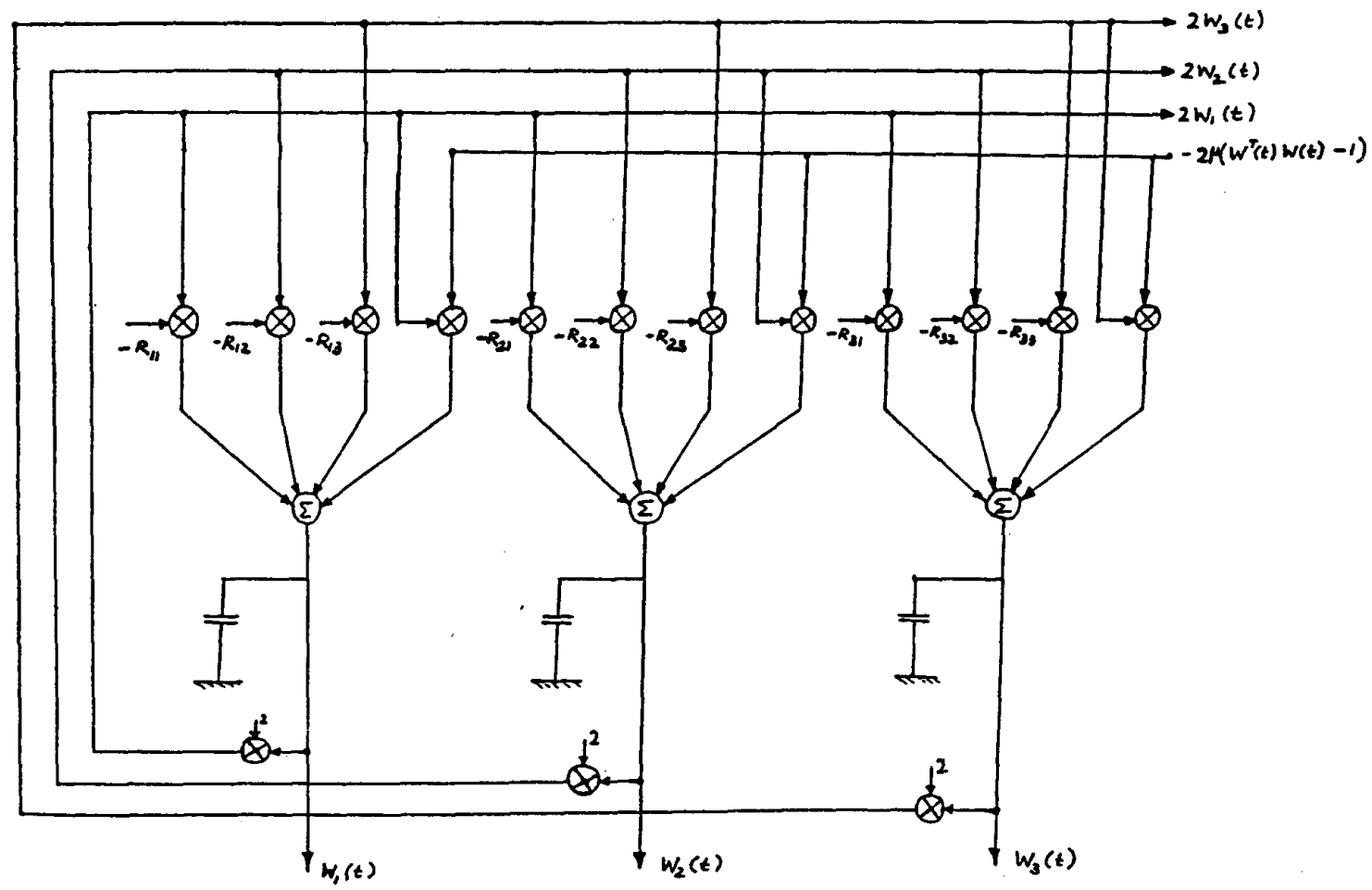

Fig.1 Neural network structure for seeking the minimum eigenvector of $\mathbf{B}(N=3, P=1)$ 